\title{
On the Dynamic Dielectric Behaviour of $\left(\mathrm{CH}_{3} \mathrm{NH}_{3}\right)_{3} \mathrm{Sb}_{2} \mathrm{Br}_{9}(\mathrm{MABA})$
}

\author{
Czesław Pawlaczyk and Ryszard Jakubas ${ }^{\text {a }}$ \\ Institute of Molecular Physics, Polish Academy of Sciences, \\ Smoluchowskiego 17, 60-179 Poznań, Poland \\ ${ }^{\text {a }}$ Faculty of Chemistry, University of Wrocław, F. Joliot-Curie 14, 50383 Wrocław, Poland \\ Reprint requests to Prof. R. J.: Fax: +48 71328 2348; E-mail; rj@wchuwr.chem.uni.wroc.pl
}

Z. Naturforsch. 58a, 189 - 193 (2003); received December 2, 2002

The complex electric permittivity of ferroelectric $\left(\mathrm{CH}_{3} \mathrm{NH}_{3}\right)_{3} \mathrm{Sb}_{2} \mathrm{Br}_{9}$ (MABA) single crystals has been measured in the frequency range $1 \mathrm{kHz}-3 \mathrm{GHz}$ between 15 and $300 \mathrm{~K}$. The dynamic dielectric behaviour of MABA is determined by the properties of high frequency relaxation of Cole-Cole type. It is thermally activated and characterised by a relatively small activation energy. The phase transitions at 168 and $134 \mathrm{~K}$ influence the amplitude of the relaxation $(\Delta \varepsilon)$ without any important changes in the relaxation frequency.

Key words: Dielectric Response; Phase Transitions; Ferroelectrics.

\section{Introduction}

The alkylammonium halogenoantimonates(III) and bismuthates(III) crystals of the general formula $\mathrm{R}_{\mathrm{a}} \mathrm{M}_{\mathrm{b}} \mathrm{X}_{3 \mathrm{~b}+\mathrm{a}}(\mathrm{R}=$ organic cation, $\mathrm{M}=\mathrm{Sb}$ or $\mathrm{Bi}, \mathrm{X}=$ $\mathrm{Cl}, \mathrm{Br}, \mathrm{I})$ form various structures, mainly with an anionic sublattice built by $\mathrm{MX}_{6}$ octahedra sharing corners, edges or faces [1-2]. Special attention attracts the $\mathrm{R}_{3} \mathrm{M}_{2} \mathrm{X}_{9}$ subclass of crystals because of their interesting ferroic properties. Several crystals having such stoichiometry exhibit ferroelasticity and ferroelectricity [3-10]. It has turned out that ferroelectric properties appear only for crystals having two-dimensional layers of polyanionic $\mathrm{M}_{2} \mathrm{X}_{9}^{3-}$ units. The structural phase transitions found in these crystals are governed by the ordering of organic cations. $\left(\mathrm{CH}_{3} \mathrm{NH}_{3}\right)_{3} \mathrm{Sb}_{2} \mathrm{Br}_{9}$ (MABA) crystallizes at room temperature with trigonal symmetry (space group $\mathrm{P} \overline{3} \mathrm{ml}$ ), similarly as its bismuth analogue $\left(\mathrm{CH}_{3} \mathrm{NH}_{3}\right)_{3} \mathrm{Bi}_{2} \mathrm{Br}_{9}$ (MABB) $[11,12]$. In spite of the fact that both methylammonium crystals are isomorphous at room temperature, they reveale a different sequence of phase transitions:

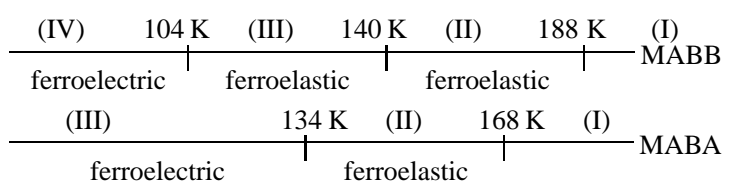

The polar phase (III) of MABA is characterised by quite small spontaneous polarization of the order of
$1.5 \cdot 10^{-3} \mathrm{C} \cdot \mathrm{m}^{-2}$ along the a-axis of the trigonal phase (I). Brillouin studies showed that the phase transition at $168 \mathrm{~K}$ is driven by an order-disorder relaxation mode $\left(\mathrm{C}_{66}\right)$ and is induced at the $\Gamma$-point of the room temperature phase [13].

The motional state of the methylammonium cations in different phases of MABA has been determined recently by means of ${ }^{1} \mathrm{H}$ and ${ }^{2} \mathrm{D}$ NMR measurements [14]. The high temperature phase (I) is characterized by isotropic rotation of two different types of methylammonium cations. The I $\rightarrow$ II phase transition is connected with the onset of freezing off the motion along the $\mathrm{C}-\mathrm{N}$ axis of all methylammonium cations (three types). Over the intermediate phase (II), during cooling, the freedom of motions of methylammonium cations is continuously diminished. Just below the II $\rightarrow$ III phase transition the $\mathrm{C}-\mathrm{N}$ axis is fixed, whereas the $\mathrm{C}_{3}$ type of motion of $\mathrm{NH}_{3}$ and $\mathrm{CH}_{3}$ groups still takes place. The change in the dynamical state of the methylammonium cations is also reflected by the dielectric response of this crystal. The freezing of the rotational motion of the cations in the $\mathrm{C}-\mathrm{N}$ axis at 168 and $134 \mathrm{~K}$ leads to a step-wise decrease of the electric permittivity when the phase transition is crossed [12].

In this paper the properties of the high frequency contribution of the dielectric relaxation (up to $3 \mathrm{GHz}$ ) in the vicinity of the 168 and $134 \mathrm{~K}$ phase transitions of MABA crystals are presented. The mechanism of the phase transitions is discussed. 


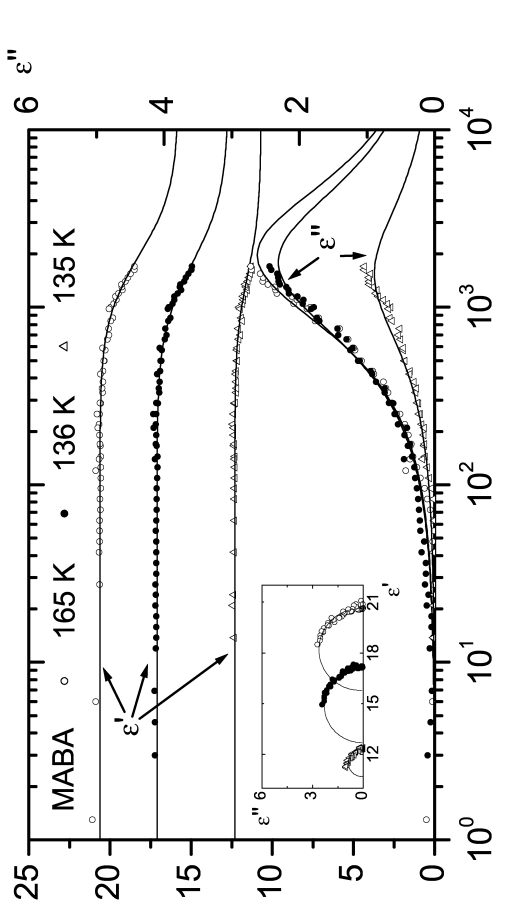

$-\omega$
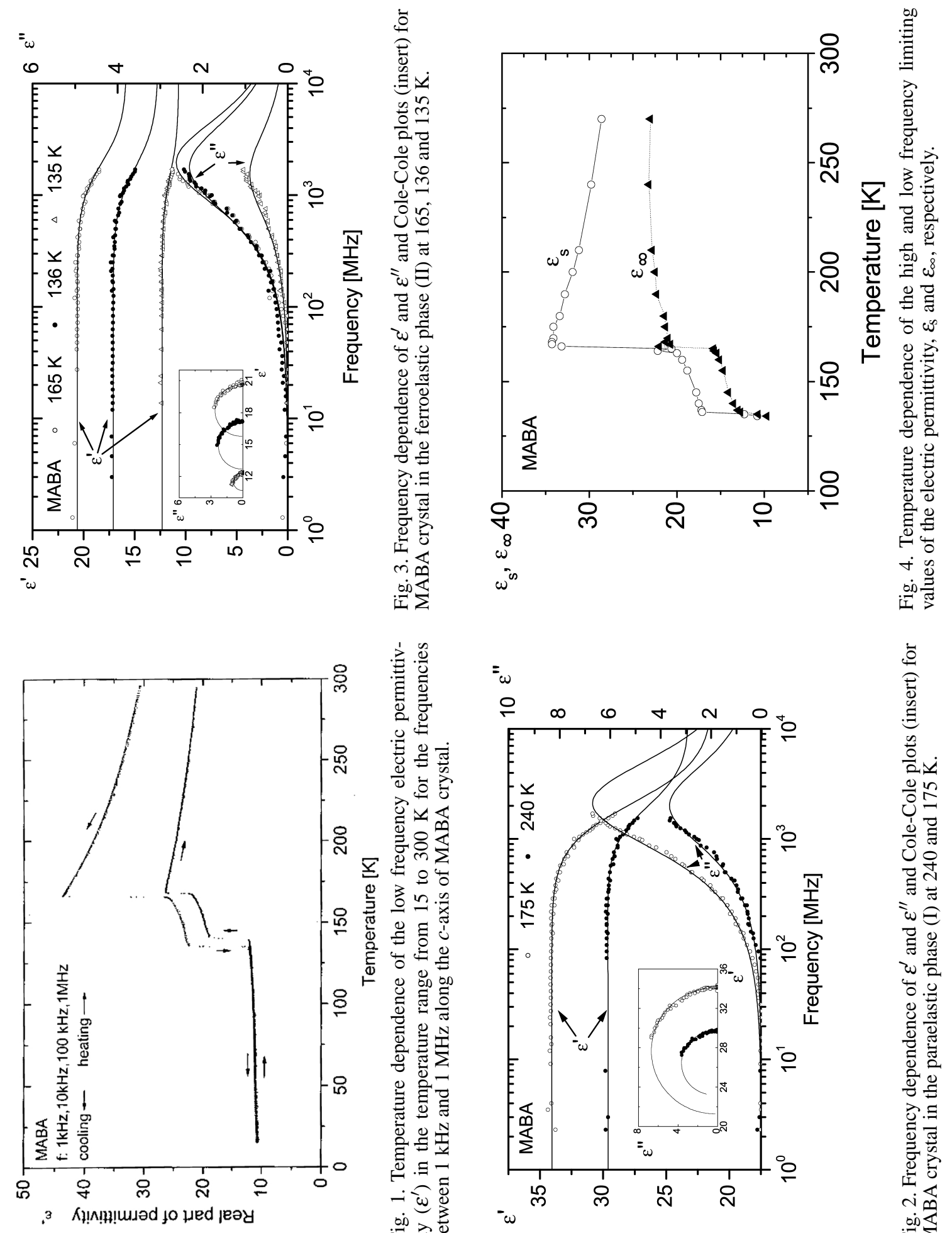

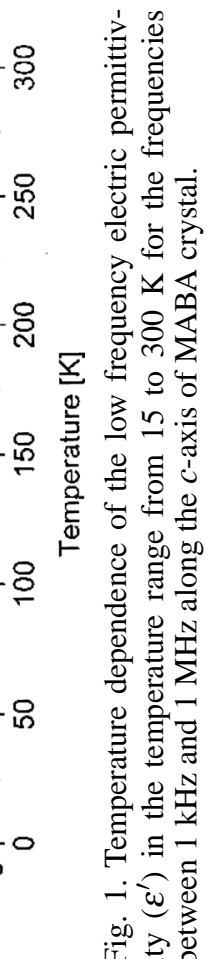

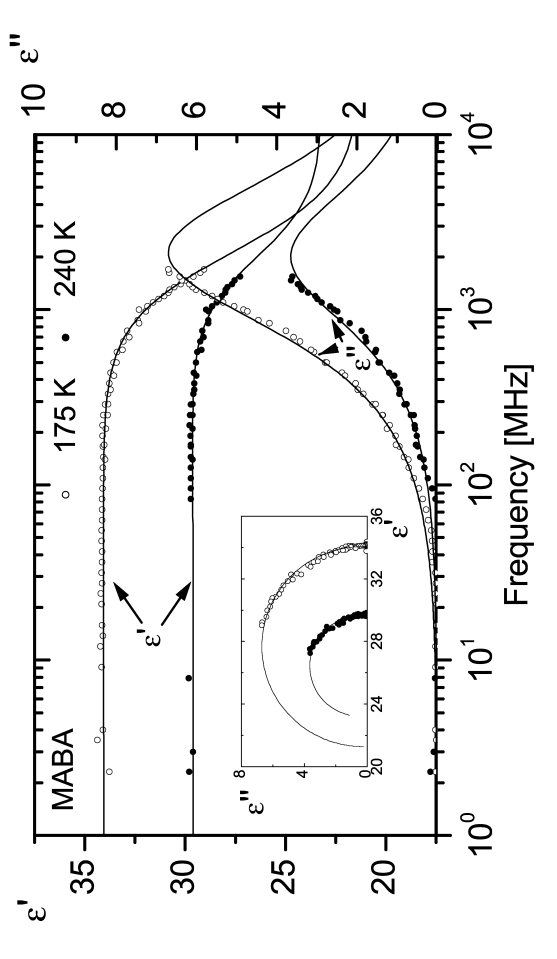

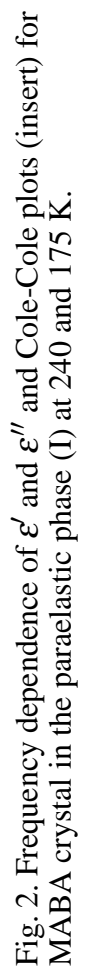




\section{Experimental}

Single crystals of $\left(\mathrm{CH}_{3} \mathrm{NH}_{3}\right)_{3} \mathrm{Sb}_{2} \mathrm{Br}_{9}$ (MABA) were grown by slow evaporation of an aqueous solution containing 3:2 mole fractions of $\mathrm{CH}_{3} \mathrm{NH}_{2}$ and $\mathrm{SbBr}_{3}$ with concentrated $\mathrm{HBr}$. Yellow and transparent crystals in the form of hexagonal plates were obtained.

The dielectric measurements were done on discs of 2-4 mm diameter and $0.5-1 \mathrm{~mm}$ thickness with the symmetry axis of the disc parallel to the $c$ axis of the MABA crystal. Dielectric measurements in the frequency range $100 \mathrm{~Hz}-1 \mathrm{MHz}$ were done using an $\mathrm{HP} 4575 \mathrm{~A} \mathrm{LCR}$ meter. In the frequency range $1 \mathrm{MHz}-$ $3 \mathrm{GHz}$ the complex dielectric permittivity was estimated by measuring the reflection factor in the coaxial line, using HP 4191A and HP 8510B impedance analysers. The equipment assembled and many times used for dielectric measurements of various ferroelectrics and other crystals at the University of Saarbrücken has been described in [15]. The temperature during the collection of the spectra was stabilised with an accuracy of at least $0.005 \mathrm{~K}$.

\section{Result and Discussion}

Figure 1 shows the temperature dependence of the real part of the complex electric permittivity measured along the $c$-axis in the frequency range $1 \mathrm{kHz}-$ $1 \mathrm{MHz}$ during cooling and heating cycles between 15 and $300 \mathrm{~K}$. It is clearly seen that no dielectric anomaly is visible from the liquid nitrogen temperature down to $15 \mathrm{~K}$. The lower value of the electric permittivity on heating (above the III $\rightarrow$ II phase transition) in comparison to that observed on cooling is probably connected with the fact that the ferroelastic- ferroelectric phase transition at $134 \mathrm{~K}$ is accompanied by a drastic splitting of single crystals parallel to the cleavage plane (001). These measurements showed that no dielectric dispersion at frequencies between $1 \mathrm{kHz}$ and $1 \mathrm{MHz}$ along the crystallographic c-axis of the trigonal room temperature phase in the whole studied temperature range is visible. In our opinion the contribution of the ferroelectric domain walls to the electric permittivity in the vicinity of $134 \mathrm{~K}$ is expected to appear below $1 \mathrm{kHz}$.

Figure 2 shows the real $\left(\varepsilon^{\prime}\right)$ and imaginary part $\left(\varepsilon^{\prime \prime}\right)$ of the complex electric permittivity in the paraelectric (simultaneous paraelastic) phase at 240 and $175 \mathrm{~K} v s$. frequency with the Cole-Cole diagrams as an insert. Figure 3 displays $\varepsilon^{\prime}$ and $\varepsilon^{\prime \prime} v s$. frequency in the ferroelastic phase (II) at 165,136 and $135 \mathrm{~K}$. The re- sults indicate that the dielectric relaxation in MABA can be well represented by a monodispersive process in a wide temperature region. Figures 2 and 3 indicate that the dielectric relaxation is almost symmetric in nature. Therefore the Cole-Cole relation

$$
\varepsilon^{*}=\varepsilon_{\infty}+\frac{\varepsilon_{\mathrm{s}}-\varepsilon_{\infty}}{1+\left(j f / f_{\mathrm{r}}\right)^{1-h}}
$$

was used to describe the dielectric response, where $f$ denotes the measuring frequency, $f_{\mathrm{r}}$ the relaxation frequency of the relaxator, $\varepsilon_{\mathrm{s}}$ and $\varepsilon_{\infty}$ are limiting values of the permittivity for the low and high frequency region $\left(\Delta \varepsilon=\left(\varepsilon_{\mathrm{s}}-\varepsilon_{\infty}\right)\right.$ is the amplitude of the relaxator), and the parameter $h$ characterised the distribution of the relaxation time. The solid lines in Figs. 2 and 3 represent the fits to the Cole-Cole function given by (1). Over the paraelectric phase a continuous increase in the relaxation strength $\Delta \varepsilon$ is visible on approaching the $168 \mathrm{~K}$ phase transition. At the transition points a step-wise decrease in the $\Delta \varepsilon$ is observed without any important change in the relaxation frequency $\left(f_{\mathrm{r}}\right)$. Figure 4 presents the temperature behaviour of the static and high frequency limiting values of $\varepsilon$ as fitting parameters. Below 168, over the ferroelastic phase (II), $\Delta \varepsilon$ is practically constant. Fig. 5 presents the $\log f_{\mathrm{r}} v s$. $T^{-1}$ plot between 130 and $300 \mathrm{~K}$. It was found that relaxation frequency of the relaxation process follows the Arrhenius relation $f_{\mathrm{r}}=f_{\mathrm{o}} \exp (-\Delta U / k T)$, giving a quite small value of energy barrier $(U=0.008 \mathrm{eV})$ in the intermediate phase (II). Unexpectedly, in the high temperature phase (I) the estimated energy barrier is practically zero. On the other hand, just below the phase transition at $134 \mathrm{~K}$ the energy barrier tends to increase distinctly. However, we have not enough experimental points to estimate it.

It is interesting (see Fig. 4) that $\varepsilon_{\infty}$ in the phases (I) and (II) is still relatively large. Taking into account that the observed dielectric ralaxator revealed its monodispersive nature, an additional dielectric relaxator should appear at frequencies higher than several dozen $\mathrm{GHz}$. The suggested relaxator may be characterised by a dielectric strength $(\Delta \varepsilon)$ of the order of 10 units. On the other hand, we can not exclude the possibility that the enhanced electric permittivity $\varepsilon_{\infty}$ may be explained in terms of the "displacive" contribution especially in the vicinity of para-ferroelectric phase transition at $134 \mathrm{~K}$. Such a situation is encountered in the closely related compound $\left(\mathrm{CH}_{3} \mathrm{NH}_{3}\right)_{3} \mathrm{Bi}_{2} \mathrm{Br}_{9}$ (MABB). The low frequency dielectric relaxation in $\mathrm{MABB}$, found around the para-ferroelectric phase transition at $104 \mathrm{~K}$ cor- 


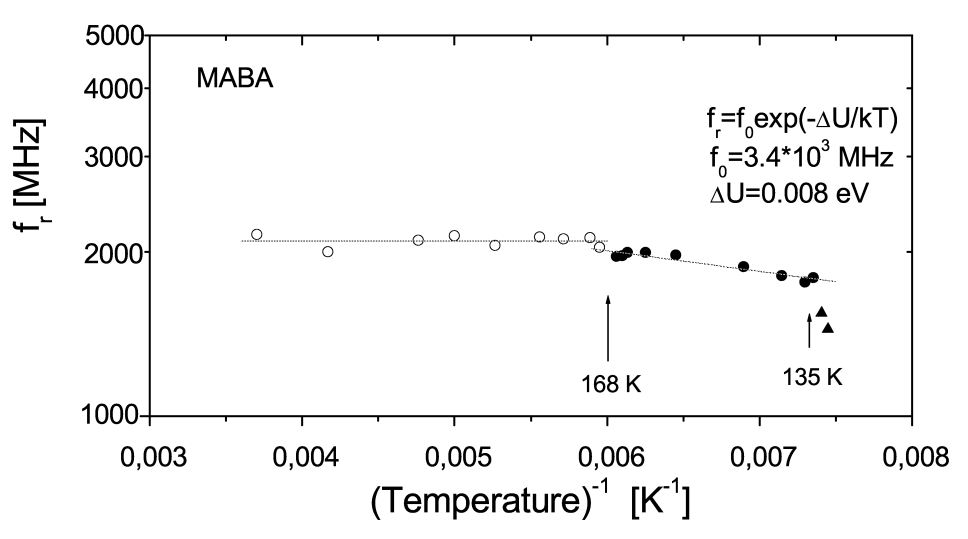

Fig. 5. Arrhenius plot of the relaxation frequency $f_{\mathrm{r}}$ over the paraelastic (I) and ferroelastic phase (II). responding to the one at $134 \mathrm{~K}$ in $\mathrm{MABA}$, did not exhibit Debey-like behaviour, and its mechanism was suggested to be of the displacive type.

The dynamic dielectric behaviour of other methylammonium halogenoantimonate(III) crystals having the same stoichiometry, e.g. $\left(\mathrm{CH}_{3} \mathrm{NH}_{3}\right)_{3} \mathrm{Sb}_{2} \mathrm{I}_{9}$ (MAIA), resembles that found in the title crystal [16]. The single relaxator found in MAIA over a wide temperature region both above and below the $147 \mathrm{~K}$ phase transition point showed a comparable relaxation frequency $f_{\mathrm{r}}$ as that estimated for MABA. Similarly as in case of MABA crystals, the dielectric relaxator in MAIA does not show a critical behaviour at $147 \mathrm{~K}$ without even noticeable changes in $f_{\mathrm{r}}$ when the phase transition is crossed.

The comparison of dynamic dielectric properties of MABA, MABB and MAIA leads to the conclusion that all methylammonium halogenoantimonate(III) and halogenobismuthate(III) crystals are characterised by similar dynamics of the methylammonium cations. The mechanism of phase transitions seems to be rather complex and the "order-disorder" and "displacive" contribution should be taken into account.

It seems interesting to mention the dielectric properties of closely related ferroelectrics having the same stoichiometry like $\left[\left(\mathrm{CH}_{3}\right)_{2} \mathrm{NH}_{2}\right]_{3} \mathrm{Sb}_{2} \mathrm{Cl}_{9}$ [17], $\left[\left(\mathrm{CH}_{3}\right)_{2} \mathrm{NH}_{2}\right]_{3} \mathrm{Sb}_{2} \mathrm{Br}_{9}$ [18], and $\left[\left(\mathrm{CH}_{3}\right)_{3} \mathrm{NH}\right]_{3} \mathrm{Sb}_{2} \mathrm{Cl}_{9}$

[1] R. Jakubas and L. Sobczyk, Phase Transitions 20, 163 (1990).

[2] M. Bujpk and J. Zaleski, Crystal Eng. 4, 241 (2001).

[3] V. Varma, R. Bhattacharjee, H. N. Vasan, and C. N. R. Rao, Spectrochim. Acta 48A, 1631 (1992).

[4] H. Ishihara, K. Watanabe, A. Iwata, K. Yamada, Y. Kinoshita, T. Okuda, V. G. Krishnan, S. Dou, and Al. Weiss, Z. Naturforsch. 47a, 65 (1992).
$[19,20]$. The dynamic dielectric properties of these crystals were determined by the presence of two independent relaxators. The low frequency relaxator revealed a critical slowing down close to $T_{\mathrm{c}}$, whereas the high frequency one was only thermally activated, showing only a subtle change in the relaxation frequency at $T_{\mathrm{c}}$. The latter relaxator possesses the same features as those found in a case of the high frequency relaxator MABA crystal.

\section{Conclusion}

(i) The dielectric properties of MABA in the highfrequency range from $1 \mathrm{MHz}$ to $3 \mathrm{GHz}$ are described by the presence of a single Cole-Cole type relaxator in the phases (I) and (II).

(ii) The relaxation frequency of the dielectric relaxator fulfils the Arrhenius relation with a relatively small activation energy, which changes insignificantly on passing through the $(\mathrm{I} \rightarrow \mathrm{II})$ phase transition at $168 \mathrm{~K}$.

(iii) The amplitude of the relaxator $(\Delta \varepsilon)$ changes stepwise at the phase transition points (at 168 and $134 \mathrm{~K}$ ) as expected from the low-frequency dielectric measurements.

(iv) Both the "order-disorder" and "displacive" contributions are suggested to appear in the phase transition mechanism at $134 \mathrm{~K}$.

[5] M. Iwata, M. Eguchi, Y. Ishibashi, S. Sasaki, H. Shimizu, T. Kawai, and S. Shimanuki, J. Phys. Soc. Japan 62, 3315 (1993).

[6] S. Ishimaru, K. Suzuki, and R. Ikeda, J. Phys. Soc. Japan 64, 1754 (1995).

[7] T. Kawai, A. Ishii, T. Kitamura, S. Shimanuki, M. Iwata, and Y. Ishibashi, J. Phys. Soc. Japan 65, 1464 (1996). 
[8] K. Gesi, M. Iwata, and Y. Ishibashi, J. Phys. Soc. Japan 65, 14 (1996).

[9] T. Kawai, E. Takao, S. Shimanuki, M. Iwata, A. Miyashita, and Y. Ishibashi, J. Phys. Soc. Japan 68, 2848 (1999).

[10] M. Iwata, A. Miyashita, H. Orihara, Y. Ishibashi, M. H. Kuok, Z. L. Rang, and S. C. Ng, Ferroelectrics 229, 233 (1999).

[11] M. Iwata and Y. Ishibashi, Ferroelectrics 135, 283 (1992).

[12] Y. Iwata, N. Koyano, M. Machida, M. Iwata, and Y. Ishibashi, Ferroelectrics 237, 229 (2000)

[13] M. H.,Kuok, S. G. Ng, L. S. Tan, Z. L. Rang, M. Iwata, and Y. Ishibashi, Solid State Commun. 108, 159 (1998).
[14] W. Medycki, Solid State NMR 14, 137 (1999).

[15] Cz. Pawlaczyk, K. Planta, Ch. Bruch, J. Stephan, and H.-G. Unruh, J. Phys.: Condens. Matter. 4, 2687 (1992).

[16] Cz. Pawlaczyk, R. Jakubas, and H.-G. Unruh, Solid State Commun. 108, 247 (1998).

[17] G. Bator and R. Jakubas, Phys. Status Solidi A 147, 591 (1995).

[18] J. Zaleski, Cz. Pawlaczyk, R. Jakubas, and H.-G. Unruh, J. Phys.: Condens. Matter 12, 7509 (2000).

[19] G. Bator, R. Jakubas, J. Zaleski, and J. Mróz, J. Appl. Phys. 88, 1015 (2000).

[20] R. Sobiestianskas, Z. Czapla, and J. Grigas, Phys. Status Solidi A 130, K69 (1992). 